\title{
Sputtering of Benzene Sample by Large Ne, Ar and Kr Clusters - Molecular Dynamics Computer Simulations
}

\author{
L. RzezniK ${ }^{a, *}$, R. PARUCH ${ }^{b}$, B.J. GARRISON ${ }^{c}$ AND Z. POSTAWA ${ }^{b}$ \\ ${ }^{a}$ University of Information Technology and Management, Rzeszów, Poland \\ ${ }^{b}$ Institute of Physics, Jagiellonian University, Kraków, Poland \\ ${ }^{c}$ Department of Chemistry, The Pennsylvania State University, PA 16802, USA
}

\begin{abstract}
Molecular dynamics simulations are employed to probe the role of an impact angle on emission efficiency of organic molecules sputtered from benzene crystal bombarded by $15 \mathrm{keV} \mathrm{Ne} 2953, \mathrm{Ar}_{2953}$, and $\mathrm{Kr}_{2953}$ clusters. It is found that both the cluster type and the angle of incidence have significant effect on the emission efficiency. The shape of the impact angle dependence does not resemble the dependence characteristic for medium size clusters $\left(\mathrm{C}_{60}, \mathrm{Ar}_{366}\right)$, where sputtering yield only moderately increases with the impact angle, has a shallow maximum around $40^{\circ}$ and then decreases. On the contrary, for the large projectiles $\left(\mathrm{Ne}_{2953}, \mathrm{Ar}_{2953}\right.$, and $\left.\mathrm{Kr}_{2953}\right)$ the emission efficiency steeply increases with the impact angle, has a pronounced maximum around $55^{\circ}$ followed by rapid signal decay. It has been found that the sputtering yield is the most sensitive to the impact angle change for $\mathrm{Kr}$ cluster projectiles, while change of the impact angle of Ne projectile has the smallest effect on the efficiency of material ejection.
\end{abstract}

DOI: 10.12693/APhysPolA.123.825

PACS: 68.49.Sf, 82.80.Ms, 83.10.Rs, 61.82.Pv, 79.20.Rf

\section{Introduction}

Gas-cluster-ion-beam (GCIB) composed of Ar atoms has become a popular ion source in time-of-flight secondary ion mass spectrometry (SIMS) since it has been shown that they allow collecting successful depth profiles in several cases where smaller cluster projectiles have failed [1]. However, still not much is known about processes occurring during Ar cluster bombardment of organic materials. It has been reported recently that the shape of the impact angle dependence of sputtering yield changes significantly with the size of the projectile bombarding organic samples and for large projectiles exhibits the shape known from the studies of atomic projectile bombardment [2-4]. Similar observation has been recently made in experiments with $\mathrm{Ar}_{2000}$ on polymer samples [5]. It has been proposed that a "washing out" mechanism is responsible for the observed phenomenon [2-4].

In this study we investigate the effect of a projectile type on the shape of the impact angle dependence of the sputtering yield of benzene molecules. This goal is accomplished by bombardment of a coarse-grained benzene crystal with $15 \mathrm{keV} \mathrm{Ne}{ }_{2953}, \mathrm{Ar}_{2953}$, and $\mathrm{Kr}_{2953}$ projectiles.

\section{Model}

Details of the MD computer simulations used to model cluster bombardment are described elsewhere [6]. The model approximating the benzene crystal consists of 307366 molecules arranged in a hemispherical sample of

*corresponding author; e-mail: lrzeznik@wsiz.rzeszow.pl

the radius $26 \mathrm{~nm}$. A coarse-grained approximation is used to model the benzene solid. This technique has proven to significantly decrease simulation time while giving results similar to the data obtained with a full atomistic model [7]. Each coarse-grained benzene molecule is represented by six $\mathrm{CH}$ particles with the mass of 13 amu. A Lennard-Jones potential is used to describe the interaction of $\mathrm{CH}-\mathrm{CH}$ particles located in different molecules. The $\mathrm{CH}-\mathrm{CH}$ interaction inside a single benzene molecule is described by a Morse potential. Details of a coarse-grained method and appropriate values for the Lennard-Jones and Morse potential parameters can be found elsewhere [7]. The interactions between Ne, $\mathrm{Ar}$, and $\mathrm{Kr}$ atoms in the projectile and between the projectile atoms and all other particles in the system are described by the Lennard-Jones potential splined with the $\mathrm{KrC}$ potential to properly describe high-energy collisions [8]. The $15 \mathrm{keV} \mathrm{Ne} 2953, \mathrm{Ar}_{2953}$, and $\mathrm{Kr}_{2953}$ projectiles are used to bombard the crystal with an impact angle changing from $0^{\circ}$ to $75^{\circ}$.

\section{Results and discussion}

The dependence of the total sputtering yield of benzene molecules on the impact angle is shown in Fig. 1 for $15 \mathrm{keV} \mathrm{Ne}{ }_{2953}, \mathrm{Ar}_{2953}, \mathrm{Kr}_{2953}$, and $\mathrm{Ar}_{60}$ projectiles. Several observations can be made from this figure. Firstly, the shapes of the distributions recorded for all large projectiles are similar. The sputtering yield steeply increases with the impact angle, has a maximum around $55^{\circ}$, and decreases above $60^{\circ}$. Similar behaviour was reported in the studies with atomic bombardment [9]. The physics is, however, different. For the $\mathrm{keV}$ atomic projectile the sputtering yield is mainly affected by the depth of primary energy deposition. In this case, most of the pri- 
mary energy is deposited below critical depth and cannot contribute to particle ejection. Increase of the impact angle shifts the profile of deposited energy closer to the surface, which enhances the sputtering yield until when back reflection of the primary energy becomes important and the yield decreases again. On the other hand, keV cluster projectile deposits its primary energy in the volume that can effectively contribute to sputtering even at angles near $0^{\circ}$. As a result, the sputtering yield can only slightly benefit from the modification of energy deposition profile. Consequently, the resulting distribution should be rather flat over a wide range of angles and then decreases when back reflection of the primary energy starts to dominate as it was shown in Fig. 1 for $\mathrm{Ar}_{60}$. Such behaviour was also observed for $\mathrm{C}_{60}[10]$ and medium size $\operatorname{Ar}_{n}[2-4,11,12]$ projectiles.

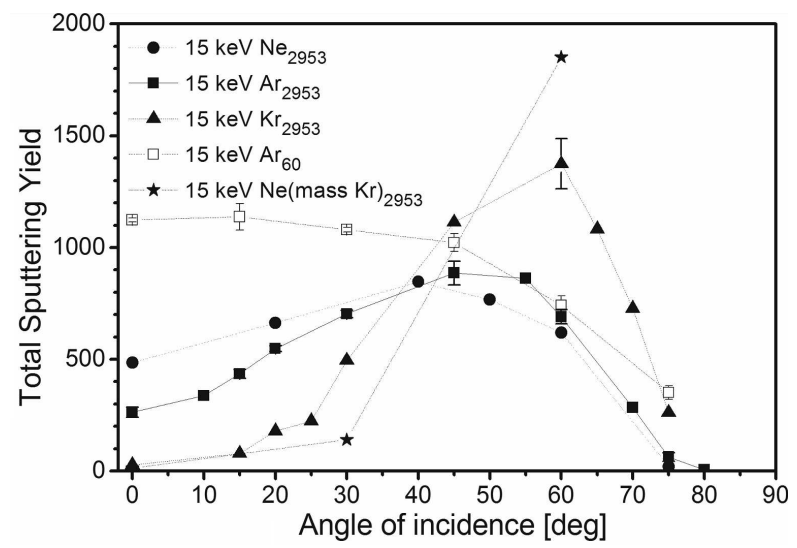

Fig. 1. Dependence of the total sputtering yield on the impact angle for bombardment of $15 \mathrm{keV}$ clusters (see the legend) of benzene crystal. Lines are drawn to guide the eye.

Surprisingly, the impact angle dependence of the sputtering yield is quite different in the case of large keV projectiles. The observed discrepancies cannot be explained by deposition of the primary energy below the critical depth. Each constituent atom of a large cluster projectile carries lower kinetic energy compared to a smaller projectile with the same total kinetic energy, which also means that it will deposit its energy closer to the surface. Also the energy back reflection is insignificant for the angles close to the surface normal, as it is shown in Fig. 2.

Mechanistic analysis of atoms movement presented in Refs. [2-4] for the $\mathrm{Ar}_{n}$ projectiles reveals that just after the impact a dense cloud of projectile atoms is formed for normal incidence bombardment, which blocks the emission of sample particle located under the impact point. On the other hand, for off normal impact, a dense flux of atoms is sliding over one side of the created crater "washing out" weakly bound benzene molecules as shown in Fig. 3.

However, while the general trend in the sputtering yield dependence on the impact angle, shown in Fig. 1, is similar for the $\mathrm{Ne}_{2953}, \mathrm{Ar}_{2953}$, and $\mathrm{Kr}_{2953}$ projectiles,

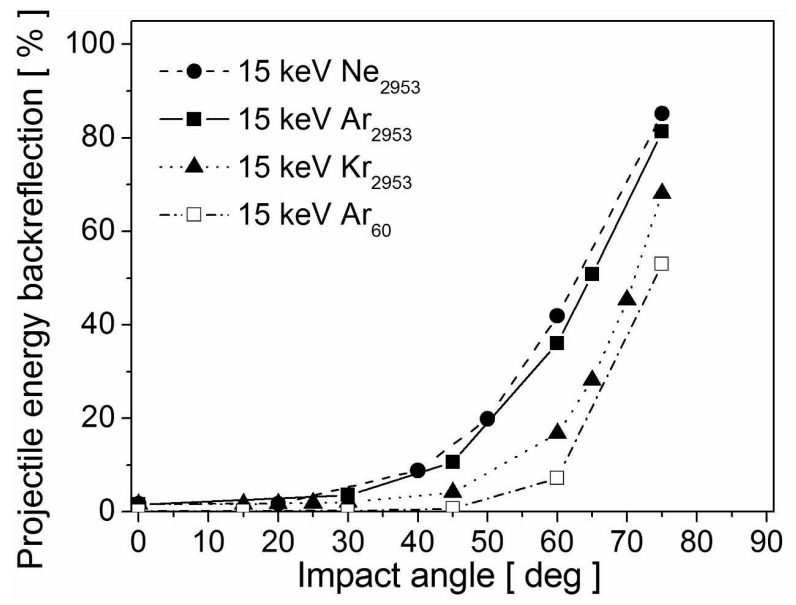

Fig. 2. Projectile energy back reflection dependence on the impact angle for benzene crystal bombardment of $15 \mathrm{keV}$ clusters (see the legend). Lines are drawn to guide the eye.

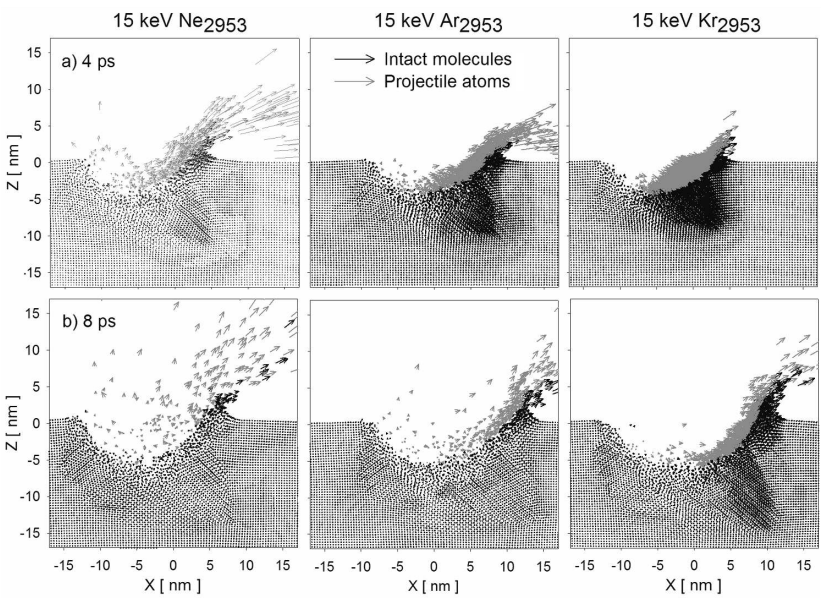

Fig. 3. Cross-sectional view, $1.5 \mathrm{~nm}$ wide, of the benzene crystal. Vectors represent the original and final positions of the center of mass of system particles at given time and time $0.5 \mathrm{ps}$ later. Intact molecules are represented by black vectors, projectile atoms are depicted by gray vectors.

there are some differences in the shape of curves plotted for each projectile. Out of these three projectiles the $\mathrm{Ne}_{2953}$ cluster stimulates the highest sputtering yield at near normal impact angles. On the other hand, at medium and large impact angles the $\mathrm{Kr}_{2953}$ cluster leads to the most efficient ejection.

The observed discrepancies can be attributed to a different mass and/or to differences in the shape of potentials describing interaction of projectile atoms. Popok et al. have shown that the range of cluster projectiles scales proportionally to the projectile momentum [13]. The momentum ratio of $\mathrm{Ne}, \mathrm{Ar}$, and $\mathrm{Kr}$ atoms is as 1:1.4:2. As a result, $\mathrm{Kr}_{2953}$ penetrates more easily through the weekly bound sample of benzene molecules 

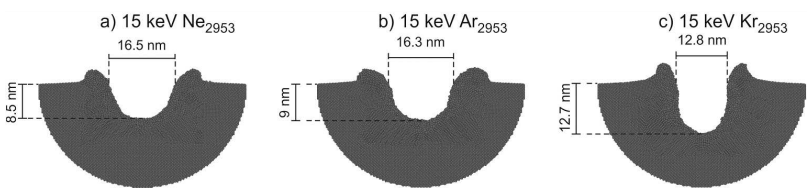

Fig. 4. Cross-sectional view, $1.5 \mathrm{~nm}$ wide, of a benzene crystal after 96 ps.

than $\mathrm{Ne}_{2953}$ or $\mathrm{Ar}_{2953}$ as presented in Fig. 4 for normal, and in Fig. 3 for off normal bombardment. Indeed, the crater created by the $\mathrm{Kr}_{2953}$ cluster is the deepest with the smallest opening for the angles close to $0^{\circ}$ (see Fig. 4). It is also horizontally more extended as compared to the craters formed by the $\mathrm{Ne}_{2953}$ and $\mathrm{Ar}_{2953}$ for off normal angles as can be seen in Fig. 3. Different craters size indicates that a different number of benzene molecules is removed from their original positions by each projectile. These molecules can be either sputtered or relocated into another location in the crystal. For normal impact angle the heaviest $\mathrm{Kr}$ projectile moves the largest number of organic particles but most of these particles are just relocated to a new position. That is why, regardless of the fact that the crater formed by this projectile is large, the sputtering yield is small. The lightest Ne projectile penetrates to the smallest depth disturbing almost twice fewer molecules than the Kr projectile. However, the relocations occur closer to the sample surface increasing the probability of ejection. As a result, the sputtering yield for the Ne projectile is seventeen times larger than for Kr. For the off normal impact angles most of moved benzene molecules are sputtered. As the number of displaced substrate particles is the smallest for the lightest Ne projectile also the sputtering yield is the lowest.

Switching from Ne to $\mathrm{Kr}$ will change not only the mass of the projectile but also will influence the shape of the potential used to describe interaction of projectile atoms. To probe which of these two factors is more important we have artificially modified mass of the Ne projectile to be the same as $\mathrm{Kr}$ without changing its interaction potentials. As it can be seen in Fig. 1, such a change indeed resulted in an impact angle dependence that is quite close to the distribution recorded for the $\mathrm{Kr}_{2953}$ projectile. This observation indicates that the atomic mass plays a more important role. Nevertheless, the influence of the interaction potential is noticeable.

\section{Conclusions}

We have investigated the effect of the projectile type on angular distributions of sputtering yield of organic molecules emitted from benzene crystal bombarded by $15 \mathrm{keV} \mathrm{Ne}{ }_{2953}, \mathrm{Ar}_{2953}$, and $\mathrm{Kr}_{2953}$ projectiles. It is shown that the projectile mass and ranges of interaction potentials can play a significant role in the case of emission efficiency, enhancing the yield for the projectiles composed of light atoms (Ne) at low angles and for the projectiles composed of heavy atoms (Kr) at off normal impact angles.

\section{Acknowledgments}

The authors would like to thank for financial support the National Centre of Science programs no. 2011/01/D/ ST4/05070, PB1839/B/H03/2011/40 and the Chemistry Division of the National Science Foundation grant no. CHE-0910564. This research was supported in part by PL-Grid Infrastructure.

\section{References}

[1] S. Ninomiya, K. Ichiki, H. Yamada, Y. Nakata, T. Seki, T. Aoki, J. Matsuo, Rapid Commun. Mass Spectrom. 23, 3264 (2009).

[2] B. Czerwinski, L. Rzeznik, R. Paruch, B.J. Garrison, Z. Postawa, Nucl. Instrum. Methods Phys. Res. B 269, 1578 (2011).

[3] Z. Postawa, R. Paruch, L. Rzeznik, B.J. Garrison, Surf. Interface Anal. 45, 35 (2013).

[4] L. Rzeznik, R. Paruch, B.J. Garrison, Z. Postawa, Surf. Interface Anal. 45, 27 (2013).

[5] D. Rading, R. Möllers, H.G. Cramer, F. Kollmer, W. Paul, E. Niehuis, Surf. Interface Anal. 45, 171 (2013).

[6] B.J. Garrison, Z. Postawa, Mass Spectrom. Rev. 27, 289 (2008).

[7] E.J. Smiley, Z. Postawa, I.A. Wojciechowski, N. Winograd, B.J. Garrison, Appl. Surf. Sci. 252 6436 (2006).

[8] R.A. Aziz, M.J. Slaman, Mol. Phys. 58, 679 (1986).

[9] R. Behrisch, W. Eckstein, Sputtering by Particle Bombardment: Experiments and Computer Calculations from Threshold to MeV Energies, Springer, Berlin 2007.

[10] K.E. Ryan, E.J. Smiley, N. Winograd, B.J. Garrison, Appl. Surf. Sci. 255, 844 (2008).

[11] L. Rzeznik, R. Paruch, B.J. Garrison, Z. Postawa, Nucl. Instrum. Methods Phys. Res. B 269, 1586 (2011).

[12] H. Kitani, N. Toyoda, J. Matsuo, I. Yamada, Nucl. Instrum. Methods Phys. Res. B 121, 489 (1997).

[13] V.N. Popok, J. Samela, K. Nordlund, E.E.B. Campbell, Phys. Rev. B 82, 201403(R) (2010). 